\title{
Relationship between the goniometric alignment and articular cartilage damage in knee osteoarthritis
}

\author{
H A Amaratunga ${ }^{1}$, S B Adikari ${ }^{1}$, T L Dassanayake ${ }^{2}$, J Gamage ${ }^{1}$, H J Suraweera ${ }^{3}$
}

\begin{abstract}
Introduction Osteoarthritis of the knee is common among the elderly. The alignment of the knee plays an important part in disease progression.

Objectives The aim was to determine the relationship between goniometric measurement of malalignment and gross and histological damage to articular cartilages of osteoarthritic knees.

Methods Anterior plane knee alignment was measured using a handheld metal goniometer in 84 patients awaiting knee replacement. Articular cartilages removed at surgery were stained and examined for macroscopic and microscopic damage which were quantified with macroscopic visual assessment score and Mankins histochemical score, respectively. The relationship between the type of knee angulation and macroscopic and microscopic damage to medial and lateral compartments was analyzed using analysis of variance (ANOVA), and the relationship between the degree of angulation and the degree of damage was determined using multiple linear regression models.

Results Varus knees had markedly higher macroscopic damage scores in the medial than the lateral compartment $(p<0.0001)$, while valgus knees showed the opposite $(p<0.0001)$. The knee angulation, together with age and BMI, predicted $54 \%$ of the macroscopic $(p<0.0001)$ and $60 \%$ of the microscopic damage $(p<0.0001)$ in varus knees.

Conclusion Anterior plane goniometric knee alignment is a significant predictor of the articular cartilage damage present in an osteoarthritic knee. Goniometric measurement is a valuable tool in assessing the disease severity as it is a simple, safe and cost-effective method.
\end{abstract}

Ceylon Medical Journal 2017; 62:167-174

DOI: http://doi.org/10.4038/cmj.v62i3.8520

\section{Introduction}

Osteoarthritis is a condition that leads to joint symptoms and signs, associated with defective integrity of articular cartilage [1]. In primary or idiopathic osteoarthritis there is no identifiable cause. Secondary osteoarthritis is caused by factor such as trauma, or inflammation.

The knee joint is most commonly affected by osteoarthritis [2]. The medial compartment of the knee transmits $60 \%-70 \%$ of the body weight and this partly explains why osteoarthritis in the medial compartment is commoner than osteoarthritis in the lateral [3, 4]. The weight distribution to each compartment of the knee is based on the angle between the femur and the tibia described as the alignment of the knee joint [5].

Malalignment of the joint is a feature of osteoarthritis and varus malalignment increases the risk of progression of medial compartment osteoarthritis whereas valgus malalignment increases the risk of progression of osteoarthritis in the lateral compartment $[6,7,8]$. Based on this we hypothesised that varus angulated knees had more damage in the medial compartment and valgus angulated knees had more damage in the lateral compartment.

Malalignment worsens with the progression of osteoarthritis [7,8]. However, measurement of knee malalignment is not carried out routinely probably because the gold standard for measurement is a full limb weight bearing radiograph [9]. This requires specialised radiographic equipment and exposes the patient to radiation. Cibere, et al report that goniometric measurement is as accurate and more feasible and matches well with the gold standard [10].

Departments of ${ }^{1}$ Anatomy and ${ }^{2}$ Physiology, Faculty of Medicine, University of Peradeniya, Sri Lanka.

${ }^{3}$ Orthopedic Unit, Teaching Hospital Peradeniya, Peradeniya, Sri Lanka.

${ }^{3}$ Orthopedic Unit, Teaching Hospital Peradeniya, Peradeniya, Sri Lanka.

Correspondence: HAA, E-mail: <himanipeiris@yahoo.com>.Received 12 December 2016 and revised version

accepted 25 Mav 2017. 
Therefore, we aimed to examine whether the degree of anterior plane knee joint malalignment measured by the goniometer correlates with the degree of macroscopic and microscopic damage to the articular cartilage of the knee.

\section{Methods}

A cross sectional study was conducted at the Orthopaedic Unit, Teaching Hospital Peradeniya a tertiary care unit draining several Provinces and the Department of Anatomy, University of Peradeniya. The orthopaedic unit carries out an average of three knee replacements a week.

A sample size of 90 was calculated on the basis that each independent variable included in a regression model (viz. age, BMI, degree of angulation) would require 15 subjects amounting to 45 subjects with each deformity condition (varus and valgus).

Sample included consecutive patients with knee osteoarthritis, scheduled for total knee replacement, who fulfilled eligibility criteria. Knee osteoarthritis was diagnosed by the consultant orthopaedic surgeon using the clinical criteria of the American College of Rheumatology [1]. Exclusion criteria were osteoarthritis secondary to rheumatoid arthritis, injury or congenital malformation, surgery or major trauma to the knee, hip or ankle.

The height and weight was measured and body mass index (BMI) was calculated [11]. Anterior plane knee alignment was measured using a handheld metal goniometer which reads the angle on the medial aspect up to $1^{0}$ [12]. Three consecutive measurements were taken by two separate investigators blinded to each other's findings. There was no significant inter or intra-observer variability. Each joint was classified as neutral, varus or valgus based on the reference ranges established for normal knee angles for this adult female population [13]. A knee angle between $179^{\circ}-183^{\circ}$ (mean $+/-2 \mathrm{SD}$ of the reference data) were classified neutral, less than $179^{\circ}$ as varus and more than $183^{\circ}$ as valgus. The degrees of deviation from $179^{\circ}$, and that from $183^{\circ}$ were considered the degree of varus and valgus malalignment, respectively.

One author conducted all knee replacements and resected the cartilage. Histopathology of the control group were obtained from normal knee joints of legs amputated due to acute trauma.

An investigator blind to the clinical details, type and degree of knee angulation examined all specimens. Macroscopic examination was done under natural light to assess the cartilage wear patterns on the tibial plateau and femoral condyles and the severity of the lesions was graded according to a published direct visual assessment scoring system (Table 1) [14]. The scores of all lesions were added to obtain a total score (maximum 20) for each compartment.

\section{Histological assessment}

Blocks $1 \mathrm{~cm} \times 1 \mathrm{~cm}$ were cut from seven predetermined areas (three from femoral condyle and four from tibial plateau) from each compartment using a fine scalpel. The 14 blocks obtained were decalcified using EDTA- $\mathrm{NH}_{4} \mathrm{OH}$ and processed [15]. Three consecutive longitudinal sections of $3 \mu \mathrm{m}$ thickness were cut from the wax blocks using a microtome (Reichert-Jung BIOCUT 2030), mounted and stained with Safranin-O-fast Green-Iron Haematoxylin [16]. Slides were examined under light microscopy and the damage to the articular cartilage was graded using the Mankins grading system [17]. Three non-overlapping fields from left to right of the tissue, at equal distance were examined and the microscopic score determined. Slides obtained from normal knees were used as reference in assessing cell density and Safranin-O staining intensity. The microscopic score for each visual field was totaled and an average score was obtained for each slide. The score of the slides from each area were averaged, and the overall microscopic score for the compartment was calculated by adding the scores from all areas sampled. The highest microscopic score achievable for a given compartment was 98 (a maximum score of 14 per area $x 7$ areas).

Ethical clearance was obtained from the Institutional Review Board, Faculty of Medicine, University of Peradeniya (No: 2008/EC/75). Written informed consent was obtained from all participants.

\section{Data analysis}

We conducted two-way analysis of variance (ANOVA) models for macroscopic and microscopic scores, with the degree of angulation (between-subject factor with three levels viz. valgus vs. neurtal vs. varus) and compartment (within subject factor with two levels viz. medial vs. lateral) as two factors. Subsequent pairwise t-tests were conducted to compare the scores between medial and lateral compartments with each type of angulation. We calculated the Pearson's correlation coefficients between the degree of angulation and damage scores of the affected compartments. We conducted multiple linear regression analyses to examine the strength of relationship between the degree of knee joint angulation and the microscopic and macroscopic damage scores of the medial compartment, adjusted for age and BMI. Given that $79.5 \%$ of the subjects had varus deformity, the regression analysis was conducted only for the subgroup with varus deformity. Since the sample with valgus deformity was small, it was 
underpowered to conduct a multiple regression analysis. SPSS statistical software (version 16.0) was used to analyse the data. The level of significance for all the statistical analyses was set at a p value of 0.05 .

\section{Results}

Out of the 98 patients (96 females) who underwent knee replacement during the study period 14 were excluded according to the exclusion criteria ( 7 had previous knee surgery, 4 had rheumatoid arthritis, 3 had osteoarthritis secondary to trauma). The sample consisted of 84 females. The age range was 46-80 years $($ mean $=64.0, \mathrm{SD}=8.5)$. Mean BMI was 27.0 $(\mathrm{SD}=4.5)$. Of the 84 knees, 66 were varus, 10 neutrally aligned and 8 were valgus. The mean angulation of the varus knees was $12.6^{0}\left(\mathrm{SD}=5.4^{0}\right.$, range: $\left.1^{0}-19^{0}\right)$, and that of the valgus knees $13.0^{\circ}\left(\mathrm{SD}=8.1^{0}\right.$, range: $7^{0}$ $20^{0}$ ).

\section{Macroscopic damage score}

Table 2 gives the average macroscopic scores. Pairwise comparisons showed that varus knees had higher macroscopic damage scores in the medial than the lateral compartment $(p<0.0001)$, while the valgus knees had markedly higher scores in the lateral than the medial compartment $(p<0.0001)$. Similar to those with varus deformity, the neutrally aligned knees showed predominant macroscopic damage to the medial compartment although the inter-compartment difference was less $(p<0.0001)$.

The degree of angulation had a significant, strong correlation with the macroscopic damage scores of the medial compartment in the varus knees $(\mathrm{r}=$ $0.730, p<0.0001)$. In the valgus knees there was significant correlation between degree of angulation and damage in the lateral compartment $(\mathrm{r}=0.796, p=$ 0.018) (Figure 1). Regression analysis which adjusted for age and BMI showed that an increase in angulation by one degree in varus knees was associated with an increase of the macroscopic damage score by 0.4 points $(p<0.0001)$. The overall regression model with varus angulation, age and BMI predicted $54.1 \%$ of the variation in the macroscopic damage (adjusted $\mathrm{R}^{2}=$ $0.541, p<0.0001)$. All varus knees with angulation more than $14^{0}$ had complete loss of articular cartilages of the medial compartment.

\section{Microscopic damage score}

Histology was done in 30 varus and all valgus and neutral specimens. Table 2 gives the average microscopic scores while figure 3 illustrates its distribution. Pairwise comparisons showed that varus knees had significantly higher microscopic damage scores in the medial than the lateral compartment $p<$ 0.0001), while the valgus knees had significantly higher scores in the lateral than the medial compartment $(p<0.0001)$. Neutrally aligned knees showed a higher microscopic score in the medial than the lateral compartment $(p=0.026)$, but the difference was not as marked as that of the varus knees.

The degree of angulation had a significant, strong correlation with the microscopic damage scores of the medial compartment in the varus knees $(\mathrm{r}=0.80$, $p<0.0001$ ) (Figure 2). The correlation was moderate in the valgus knees but not significant $(\mathrm{r}=0.53 ; p=$ $0.175)$. Regression analysis which adjusted for age and BMI showed that an increase in angulation by one degree in a varus knee was associated with an increase of the microscopic damage score by 1.5 points $(p<$ $0.0001)$. The overall regression model with varus angulation, age and BMI predicted nearly $60 \%$ of the variation in the microscopic damage (adjusted $\mathrm{R}^{2}=$ $0.594 ; p<0.0001)$. There was strong correlation between macroscopic and microscopic damage scores $(\mathrm{r}=0.789, p<0.0001)$.

\section{Discussion}

Osteoarthritis affects approximately $10 \%$ of the world's population. The knee joint is the most commonly affected $[18,19]$. Our sample consisted of females with a mean age of 64 years. The sample is thus representative of patients with primary osteoarthritis as it is reported predominantly in women aged 60 years and above [20,21]. Obesity is also a known risk factor as seen in our sample which had an average BMI of 27 [22].

We examined the relationship between goniometric measurement of knee malalignment and the macroscopic and microscopic changes to the medial and lateral compartment articular cartilages in varus, valgus and neutrally aligned knees. As described by previous groups who used radiological evidence, we found varus malalignment to damage predominantly the medial compartment and valgus malalignment to predominantly damage the lateral compartment $[5,6$, 23]. The degree of angulation was strongly correlated with the degree of damage to the corresponding compartment. In varus knees, angulation was a strong predictor of damage even after adjusting for the possible effects of age and BMI. The strong correlation indicates that goniometric measurement can be used as a reliable measure of assessing the degree of severity of joint destruction. Therefore, although knee goniometry cannot replace radiographic assessment, it adds to the assessment of severity in a clinical setting. Once malaligned, the knee alignment cannot be corrected except by surgery. However, the rate of worsening of the malalignment can be slowed by different treatment options. Measurement of 
malalignment therefore cannot be used as an indicator of the efficacy of the treatment but rather as an indicator of the underlying joint damage.

In general, varus osteoarthritic knees showed a greater degree of damage than valgus or neutrally aligned knees with osteoarthritis. In varus knees, as evidenced by the microscopic and macroscopic scores, the most severely damaged area was the medial edge of the articular cartilage on the tibial plateaus. The degree of damage becomes progressively less in the lateral areas and the lateral compartment was relatively well preserved in these knees. Neutrally aligned knees showed damage in both compartments, however the medial compartment was more severely damaged in these knees, probably due to the fact that $60-70 \%$ of the body weight is transmitted through the medial compartment [24].

The pattern was reversed in the valgus knees with the lateral edge of the tibial plateau being damaged the most. In these joints, the macroscopic and microscopic scores increased towards the lateral edge of the specimens. In the femoral condyles the damage was greatest at the inferior surface which comes into direct contact with the tibia when the knee is partially flexed and extended. A previous study that graded 42 tibial plateaus and the femoral condyles of varus and valgus knees using a simple four stage macroscopic grading system, also reports that the damage was most severe in the central peripheral medial area of varus knees and the central peripheral lateral edge in the valgus knees [25].

Our findings indicate that even one degree of change in the deformity angle significantly increases the damage to the articular cartilage, as evidenced by the parallel increase in both the macroscopic and microscopic scores. These findings are consistent with previous studies which employed radiological methods of assessment and state that a 4-5 fold increase in the odds of progression can be attributed to the degree of malalignment and that more severe the damage faster the effect of malalignment on progression [6,23]. Our study provides direct pathological evidence for the relationship between malalignment and severity of cartilage damage and further strengthens the above relationship.

In summary, our findings indicate that the goniometric measurement of the anterior plane knee joint alignment can closely predict the severity of macroscopic and microscopic articular cartilage damage present in an osteoarthritic knee. Thus we conclude that anterior plane goniometry is a simple yet sensitive tool in assessing and monitoring the severity of damage in osteoarthritic knee joints. This assessment can be incorporated into knee examination particularly in resource-limited units in Sri Lanka where a full-length anteroposterior weight bearing limb radiograph cannot be obtained.

\section{Conflict of interests}

There are no conflicts of interest.

\section{References}

1. Altman R, Asch E, Bloch D, Bole G, Borenstein D, Brandt K. Development of criteria for the classification and reporting of osteoarthritis. Classification of osteoarthritis of the knee. Diagnostic and Therapeutic Criteria Committee of the American Rheumatism Association. Arthritis Rheum 1986; 29: 1039-49.

2. Woolf AD, Pfleger B. Burden of major musculoskeletal conditions Bull World Health Organ 2003; 81: 646-56.

3. Andriacchi TP. Dynamics of knee malalignment Orthop Clin North Am 1994; 25: 395-403.

4. Ledingham J,Regan M, Jones A, Doherty $M$. Radiographic patterns and associations of osteoarthritis of the knee in patients referred to hospital Ann Rheum Dis 1993; 52: 520-6.

5. Hsu RW, Himeno S, Coventry MB, Chao EY. Normal axial alignment of the lower extremity and load-bearing distribution at the knee Clin Orthop Relat Res 1990; 255: 215-27.

6. Brouwer GM, van Tol AW, Bergink AP, et al. Association between valgus and varus alignment and the development and progression of radiographic osteoarthritis of the knee. Arthritis Rheum 2007; 56: 1204-11.

7. Sharma L, Song J, Felson DT, Cahue S, Shamiyeh E, Dunlop DD. The role of knee alignment in disease progression and functional decline in knee osteoarthritis. JAMA 2001; 286: 188-95.

8. Felson DT, Niu J, Gross KD, et al. Valgus malalignment is a risk factor for lateral knee osteoarthritis incidence and progression: findings from the Multicenter Osteoarthritis Study and the Osteoarthritis Initiative Arthritis Rheum 2013; 65: 355-62.

9. Moreland JR, Bassett LW, Hanker GJ. Radiographic analysis of the axial alignment of the lower extremity J Bone Joint Surg Am 1987; 69: 745-9.

10. Cibere J, Bellamy N, Thorne A, et al. Reliability of the knee examination in osteoarthritis: effect of standardization Arthritis Rheum 2004; 50: 458-68. 
11. Garrow JS, Webster J. Quetelet's index (W/H2) as a measure of fatness. Int J Obes 1985; 9: 147-53.

12. Kraus VB, Vail TP, Worrell T, McDaniel G. A comparative assessment of alignment angle of the knee by radiographic and physical examination methods. Arthritis Rheum 2005; 52: 1730-5.

13. Amaratunga HA, Adikari SB, Dassanayaka TL, Chandrasekara M, Suraweera HJ. Goniometric alignment of the normal knee joint and the factors which affect it. Ceylon Med J 2014; 59: 45-48.

14. Noyes FR, Stabler CL. A system for grading articular cartilage lesions at arthroscopy $\mathrm{Am} \mathrm{J}$ Sports Med 1989; 17: 505-13.

15. Sanderson C, Radley K, Mayton L. Ethylenediaminetetraacetic acid in ammonium hydroxide for reducing decalcification time Biotech Histochem 1995; 70: 12-8.

16. Yuehuei HA, Martin KL. Handbook of Histology Methods for Bone and Cartilage. Totawa. New jersey: Humana Press Inc, 2002.

17. Mankin HJ, Dorfman H, Lippiello L, Zarins A. Biochemical and metabolic abnormalities in articular cartilage from osteo-arthritic human hips. II. Correlation of morphology with biochemical and metabolic data J Bone Joint Surg Am 1971; 53: 523-37.
18. Symmons D, Mathers C, Pfleger B. World health organization. Global burden of osteoarthritis in the year 2000, 2003

http:/www.who.int/entity/healthinfo/statistics/bod osteoarthritis.pdf.

19. Woolf AD, Pfleger B. Burden of major musculoskeletal conditions. Bull World Health Organ 2003; 81: 646-56.

20. McAlindon TE, Cooper C, Kirwan JR, Dieppe PA. Determinants of disability in osteoarthritis of the knee. Ann Rheum disease 1993; 52: 258-62.

21. Felson DT, Lawrence RC, Dieppe PA, et al. Osteoarthritis: new insights. Part 1: the disease and its risk factors. Ann of Int Med 2000; 133: 635-46.

22. Dawson J, Juszczak E, Thorogood M, Marks SA, Dodd C, Fitzpatrick R. An investigation of risk factors for symptomatic osteoarthritis of the knee in women using a life course approach. $J$ EpidemiolCommunity Health, 2003; 57: 823-30.

23. Cerejo R, Dunlop DD, Cahue S, Channin D, Song $\mathrm{J}$, Sharma L. The influence of alignment on risk of knee osteoarthritis progression according to baseline stage of disease. Arthritis Rheum 2002; 46: 2632-6.

24. Andriacchi TP. Dynamics of knee malalignment. Orthop Clin North Am 1994; 25: 395-403.

25. Weidow J, Pak J, Karrholm J. Different patterns of cartilage wear in medial and lateral gonarthrosis Acta Orthop Scand 2002; 73: 326-9. 


\section{Table 1. Direct visual assessment score}

\begin{tabular}{lll}
\hline Description of lesion & Scores & Scores \\
& $10-15 \mathrm{~mm}$ sized lesion & $>15 \mathrm{~mm}$ sized lesion \\
\hline No visible lesion & 0 & 0 \\
Lesion with fissures, fragmentation- half thickness & 2 & 4 \\
Lesion with fissures, fragmentation- full thickness & 3 & 6 \\
Bone exposed & 5 & 10 \\
\hline
\end{tabular}

Table 2. Macroscopic and microscopic scores of the medial and lateral compartments of the varus, valgus and neutrally aligned knees

\begin{tabular}{clllc}
\hline $\begin{array}{c}\text { Type of } \\
\text { malalignment }\end{array}$ & \multicolumn{2}{c}{$\begin{array}{l}\text { Macroscopic score (maximum 20) Mean } \\
\text { (SD) }\end{array}$} & \multicolumn{2}{c}{$\begin{array}{c}\text { Microscopic score (maximum 98) } \\
\text { Mean (SD) }\end{array}$} \\
& $\begin{array}{l}\text { Medial } \\
\text { compartment }\end{array}$ & $\begin{array}{l}\text { Lateral } \\
\text { compartment }\end{array}$ & $\begin{array}{l}\text { Medial } \\
\text { compartment }\end{array}$ & $\begin{array}{c}\text { Lateral } \\
\text { compartment }\end{array}$ \\
Varus (n=66) & $16.5(3.1)$ & $2.1(1.1)$ & $55.3(10.5)$ & $13.8(8.6)$ \\
Neutral (n=10) & $16.7(3.3)$ & $7.1(3.2)$ & $46.7(6.3)$ & $37.9(7.07)$ \\
Valgus (n=8) & $5.6(6.6)$ & $68.3(21.1)$ & $12.2(7.7)$ & $44.1(5.02)$ \\
\hline
\end{tabular}



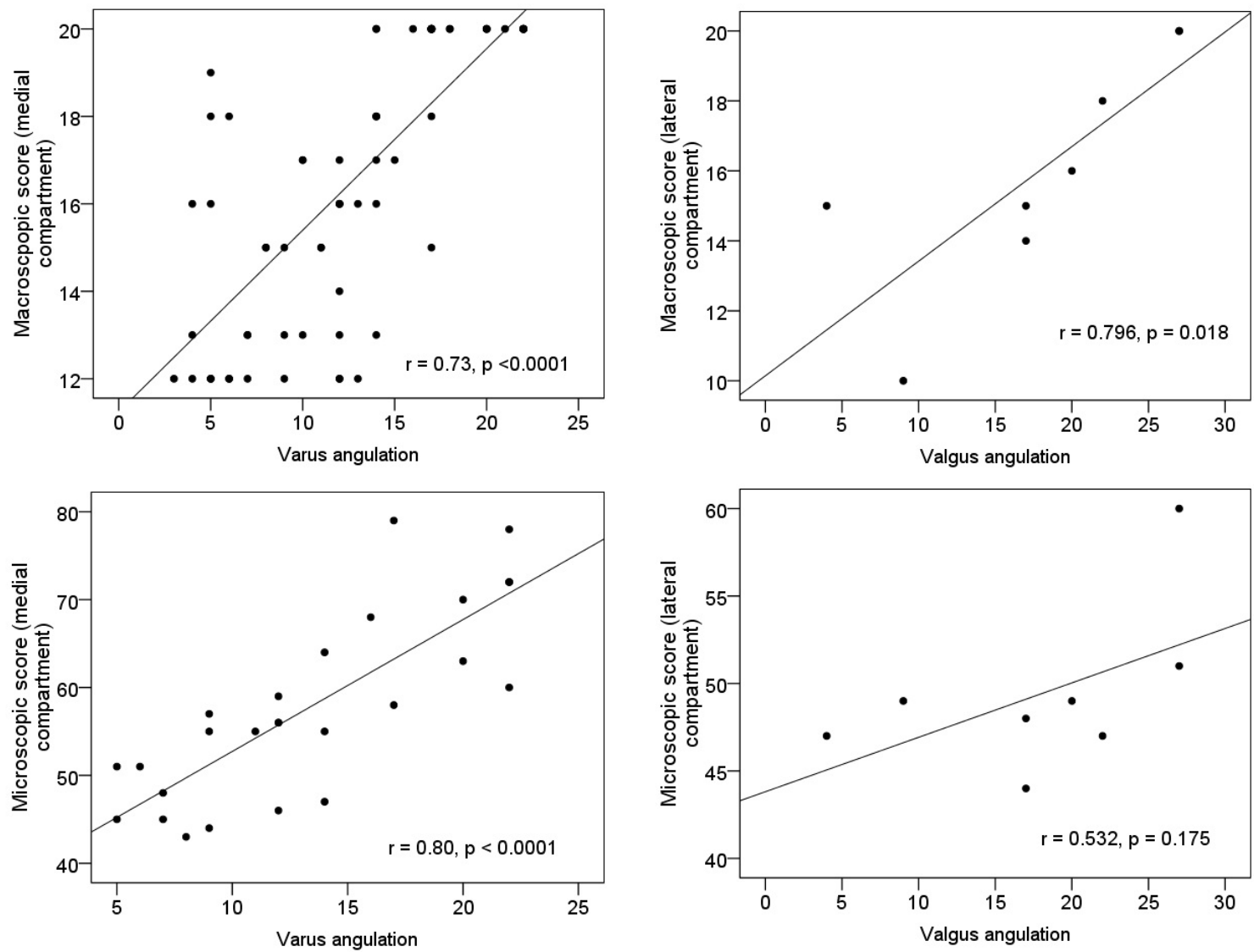

Figure 1. Correlation between the degree of malalignment and the degree of macroscopic and microscopic damage to corresponding compartments of the joint 


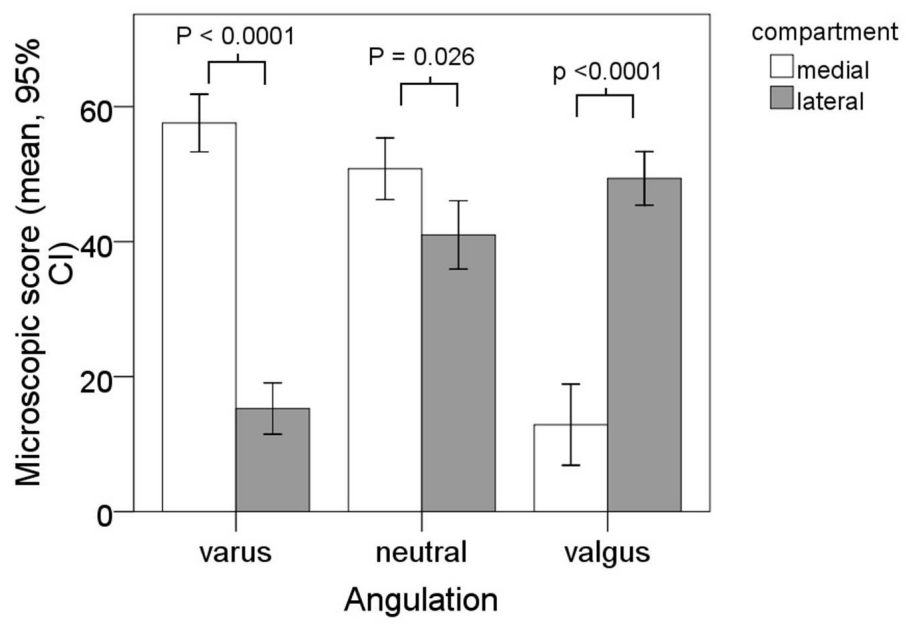

Figure 2. Microscopic damage (Mankin's) scores in medial and lateral compartments of neutral, varusand valgus malaligned knees

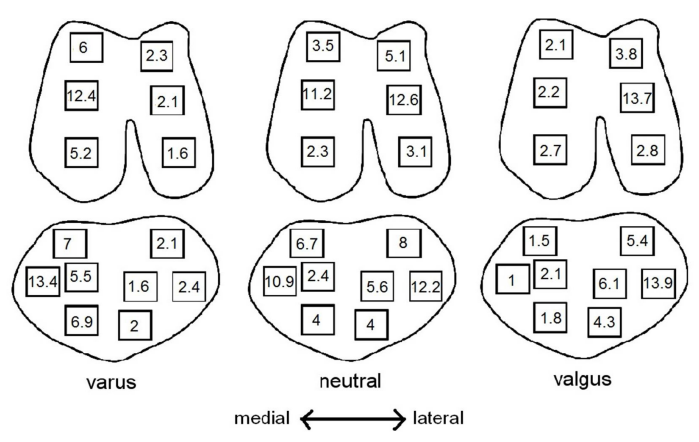

Figure 3. Distribution of mean microscopic damage scores in tibial and femoral cartilages in neutral, varus and valgus malaligned knees and source are credited. 